\title{
The EUV spectrum of the Sun: SOHO, SEM, and CDS irradiances
}

\author{
G. Del Zanna ${ }^{1}$, S. R. Wieman ${ }^{2}$, V. Andretta ${ }^{3}$, and L. Didkovsky ${ }^{2}$ \\ ${ }^{1}$ DAMTP, Centre for Mathematical Sciences, University of Cambridge, Wilberforce Road, Cambridge CB3 0WA, UK \\ e-mail: g.del-zanna@damtp.cam.ac.uk \\ 2 University of Southern California Space Sciences Center, 835 Bloom Walk, Los Angeles, CA 90089, USA \\ 3 INAF-Osservatorio Astronomico di Capodimonte, Salita Moiariello, 16, 80131 Napoli, Italy
}

Received 31 March 2015 / Accepted 19 June 2015

\begin{abstract}
We use calibrated extreme-UV (EUV) spectral irradiances obtained from observations with the Solar \& Heliospheric Observatory (SOHO) Coronal Diagnostics Spectrometer Normal Incidence Spectrometer (NIS) to estimate the signal measured by the Solar EUV Monitor (SEM) first-order band, 260 to $340 \AA$ (SEM 1). The NIS observes the resonance lines He II $304 \AA$ and Si XI $303 \AA$ directly in second order. The irradiances of the other lines in the band are estimated with a differential emission measure (DEM) modelling, using updated atomic data. The observations analysed here were obtained during 1998-2011, which means that they span the maximum and minimum of Cycle 23. The current knowledge of the SEM 1 degradation is used to find effective areas during the dates of the NIS observations and to predict the SEM 1 count rates across the band. The total count rates, estimated by folding the NIS-based spectra with the SEM 1 effective areas, agree very well (within 10-20\%) with the observed ones during solar minimum conditions, when the He II $304 \AA$ is the dominant contribution to the band. Excellent agreement with the Solar Dynamics Observatory (SDO) Extreme ultraviolet Variability Experiment (EVE) observations is also found. On the other hand, the predicted SEM 1 count rates during the Cycle-23 maximum are significantly (by about 30\%) lower than the observed ones. The solar spectrum in the SEM 1 band changes significantly during maximum conditions, with the He II $304 \AA$ only contributing about $40 \%$. A significant fraction of the observed count rates comes from coronal emission in an off-band spectral region that has recently been discovered. An explanation for the discrepancy needs further investigation.
\end{abstract}

Key words. Sun: corona - techniques: spectroscopic

\section{Introduction}

The present paper is part of an on-going effort to study the extreme-UV (EUV) spectral radiance and irradiance over the solar cycle. In Paper I (Del Zanna et al. 2010) we presented the long-term calibration of the Solar and Heliospheric Observatory (SOHO) (CDS; Harrison et al. 1995) Normal Incidence Spectrometer (NIS), while in Paper II (Del Zanna \& Andretta 2011) we presented the first measurements of the EUV spectral irradiance during a solar cycle in 1998-2010. Paper III (Andretta \& Del Zanna 2014) discussed the characteristics of the corresponding EUV radiances for the first time. This paper discusses the intercalibration of the NIS and the SOHO Solar EUV Monitor (SEM) first-order band (SEM 1), which nominally covers the 260 to $340 \AA$ spectral region. This region contains the strongest EUV solar line (the He II $304 \AA$ A resonance transition), as well as several strong coronal lines.

The SEM 1 channel indeed observes a significant fraction of the solar EUV emission, which is absorbed in the Earth's thermosphere, creating the ionosphere. The solar EUV variability causes dramatic changes in the temperature and density of the thermosphere, directly affecting satellite drag and possibly also affecting other atmospheric layers. There have been some indications of very low levels of solar activity during the extended minimum of Cycle 23/24 from around 2008 to 2010. SEM 1 observations indicated, for example, a reduction of about $12 \%$ in the EUV flux compared to the previous minimum in 1996
(Didkovsky \& Wieman 2014). However, any such measurement depends critically on a good understanding of the instrument calibration and in-flight degradation. Furthermore, the interpretation of any broad-band observation requires knowledge of the underlying solar spectrum, which only spectrometers can provide. Solar Dynamics Observatory (SDO) Extreme ultraviolet Variability Experiment (EVE, see Woods et al. 2012) observations at moderate resolution with the Multiple EUV Grating Spectrographs (MEGS) were available across a wide wavelength range from May 2010 until May 2014, when MEGSA ceased to operate. Wieman et al. (2014) compared the EVE MEGS spectra during 2010-2014 to the SEM observations. The SEM 1 (version 3) irradiances were found to be overestimated by about $20 \%$ to $25 \%$, when compared to those estimated from the SDO/EVE MEGS version 3 data. This comparison prompted a revision of the SEM 1 calibration, which is still under investigation.

The present paper extends this comparison to the period 1998 to 2010, using NIS full-Sun observations. The NIS observes the 308-379 and 513-633 $\AA$ ranges in two spectral channels (NIS 1 and 2, respectively). The He II $304 \AA$ and Si XI $303 \AA$ are observed by the NIS 2 channel in second order.

The present study is also within the SOLID ${ }^{1}$ network effort to provide the best possible solar spectral irradiances. As a first step on the modelling side, we assessed the completeness and

\footnotetext{
http://projects.pmodwrc.ch/solid/
} 
accuracy of the currently available atomic data. As a second step, we improved the CHIANTI ${ }^{2}$ database with recent atomic data (mostly produced by the UK APAP network ${ }^{3}$ ) to produce version 8 (Del Zanna et al. 2015), which is used here. Sections 2 and 3 briefly describe the CDS and SEM observations, Sect. 4 presents the results of the comparison, and Sect. 5 draws the conclusions.

\section{The CDS NIS}

From 1998 the NIS instrument has carried out full-Sun scans recording radiances in all the wavelengths. In Paper II, we described the methods used to obtain calibrated NIS irradiances from full-Sun radiance measurements during 1998-2010. We used these irradiances, plus two other observations after 2010 that we have analysed here. One was obtained on 2011 Mar 23 when a clone EVE calibration sounding rocket was flown and during a period of relatively low solar activity when the radio $10.7 \mathrm{~cm}$ flux was 104 solar flux units $(1$ s.f.u. = $\left.10^{-22} \mathrm{~W} \mathrm{~m}^{-2} \mathrm{~Hz}^{-1}\right)$. Another one was obtained during a day of increased activity (2012 Apr. 19), when the radio $10.7 \mathrm{~cm}$ flux was 139.

The long-term degradation of the NIS instrument during the 1996-2010 period as described in Paper I was obtained by assuming that the quiet Sun radiances of low-temperature lines (observed with the wide 90" slit) were constant throughout the cycle. The long-term degradation was combined with an earlier in-flight relative calibration (Del Zanna et al. 2001) and with the absolute calibration at $584 \AA$ obtained from a comparison between NIS measurements and those from a NASA/Laboratory for Atmospheric and Space Physics (LASP) EUV Grating Spectrograph (EGS) rocket, flown in 1997 May 15 (Brekke et al. 2000). Very good agreement (normally well within 30\%) was found between the calibrated NIS radiances and irradiances and those measured by several sounding rockets. One was the EUNIS sounding rocket, which measured quiet Sun radiances in 2007 (see Wang et al. 2011). Another one was the 2008 April 14 sounding rocket, which carried a prototype (PEVE, see Chamberlin et al. 2007a; Hock et al. 2009) of the SDO EVE. Very good agreement between the NIS and PEVE irradiances for most of the lines was found (Del Zanna et al. 2010), with the exception of the strongest line in the EUV, the He II $304 \AA$ resonance line. The uncertainties in the various calibration factors, together with these comparisons, suggest an overall uncertainty of $20-30 \%$ for the NIS calibration.

Del Zanna \& Andretta (2011) revised the NIS calibration for the important He II $304 \AA$ line, the most prominent in the EUV, pointing out that in most previous observations, its calibration was incorrect by large factors. Very good agreement (within 10\%) with the recent quiet-Sun He II $304 \AA$ radiances measured by EUNIS 2007 was found, as well as with the SDO PEVE irradiance.

As part of the SOLID effort, the CDS calibration was extended for the period 2010-2014. We did not find any further long-term degradation effects, but realised that the correction for the burn-in in the cores of some of the strong lines was not accurate, producing an increasing underestimate of the line radiances. This underestimate was small during the 2007-2010, but reached about $30 \%$ in 2014. The revised burn-in and long-term corrections will be described in a forthcoming paper (Del Zanna $\&$ Andretta 2015). For the present paper, it sufficies to point out

\footnotetext{
2 wWw.chiantidatabase. org

3 wWw . apap-network.org
}

that the burn-in for the He II $304 \AA$ has not been modified, and the suggested changes in the long-term degradation are only a few percent for the dates considered here and for some wavelengths, hence are negligible.

\section{The SEM 1}

The SEM instrument consists of a free-standing transmission grating and three silicon photodiode detectors, one observing the zeroth order, and the other two observing the +1 and -1 orders of diffraction (channels 1 and 3 of SEM 1). Thin-film aluminium filters were placed in front of the entrance slit and on the surface of the detectors to prevent detection of visible light. The SEM data processing algorithm for converting raw detector count rates to calibrated irradiance values is based on the NIST-measured channel spectral response functions, a time- and wavelength- dependent degradation model, and a solar reference spectrum that provides an appropriate spectral weighting of the response function and, in the case of the SEM 1 channels, a correction for sensitivity outside of the nominal 260-340 $\AA$ band pass. For further information on the SEM instrument, see references in Wieman et al. (2014).

The need for the degradation correction arises from a decrease in sensitivity that the SEM has suffered over time, which has been attributed to the build-up and subsequent UV-driven polymerization of hydrocarbon contaminant on the front filter. This degradation was discovered, and continues to be monitored, based on comparisons with irradiances measured on periodic sounding rocket flights using a SEM clone instrument and a neon rare gas ionization cell (RGIC) absolute detector. The degradation correction is based on the modelled reduction in filter transmission due to a pure carbon contaminant layer that grows in thickness with time. The function describing contaminant thickness versus time is an exponential curve with coefficients that are established (and revised as additional sounding rocket measurements are obtained) to provide the best fit between the corrected on-orbit SEM irradiances and the available set of sounding rocket measurements. Because the degradation model is based on the transmission of carbon, which decreases with increasing wavelength over the SEM 1 range of spectral sensitivity, the correction is wavelength-dependent and effectively changes the shape of the SEM response function, not only reducing overall sensitivity but also biasing it in favour of shorter wavelengths over time.

In Wieman et al. (2014) the aforementioned differences between SEM version 3.1 irradiance values and those from SDO EVE MEGS and EVE ESP were traced to inaccuracies in the SEM 1 instrument response function and in the solar reference spectrum used in data processing. Although the response function for the on-orbit SEM was measured prior to the launch of SOHO, a 2007 NIST calibration of the SEM sounding rocket clone instrument (which is nominally identical to the in-flight version) revealed that the SEM 1 sensitivity outside of its nominal 260-340 $\AA$ band pass is greater than what was measured pre-launch owing to the grazing incidence reflection off of a portion of the instrument housing that was left off for the original calibration.

SEM irradiances for Version 3.1 and earlier are determined using SOLERS22 (Woods et al. 1998), which is a single, fixed reference spectrum and thus does not represent changes in spectral distribution with solar activity. Furthermore, for wavelengths affecting the SEM 1 irradiance calculation, SOLERS22 was found to exhibit characteristic differences in spectral shape 
compared to EVE MEGS measured spectra that were evident independently of the activity level of the MEGS measurement.

In the Wieman et al. (2014) study, it was shown that reprocessing the SEM data for the interval of SOHO and SDO mission overlap using a revised response function based on the 2007 SEM clone calibration and concurrent measured reference spectra from EVE MEGS resulted in a $15 \%$ to $25 \%$ shift in the SEM irradiance values, which brought them into very good agreement with those from EVE version 2.

In Didkovsky \& Wieman (2014), the SEM 1 daily average data from the 1996 start of the mission through 2013 were reprocessed using time-dependent reference spectra and the updated SEM 1 response function. For this work, the flare irradiance spectral model (FISM; Chamberlin et al. 2007b) was used as a source of daily reference spectra since continuous spectral measurements like those from EVE MEGS are not available throughout most of the SOHO mission. All sounding rocket measurements available at the time of the study, including several additional rocket flights that occurred after the release of version 3.1, were processed with concurrent FISM spectra and the revised response function, which resulted in an updated estimate of the carbon contaminant growth curve and associated degradation correction. However, this revised assessment of SEM degradation was not found to be dramatically different from that of version 3.1. For example, for the aforementioned observations of lower EUV irradiance levels during the anomalous Solar Cycle 23/24 minimum compared to the previous minimum (which depend directly on the determined SEM degradation), an inter-minima decrease of $15 \%$ is estimated based on version 3.1 irradiances (Didkovsky et al. 2010), while the estimated decrease from the Didkovsky \& Wieman (2014) study, at $12 \%$, is only modestly different. While a discussion of the relative intensity of the two minima is not within the scope of the present paper, these results illustrate that the SEM degradation model is not substantially influenced by the revised data processing parameters.

The SDO EVE MEGS, as for all EUV instruments, has suffered significant degradation, and its calibration is continuously being revised with a combination of methods. These methods include observations with different front filters and comparisons with observations of a clone on sounding rocket flights. Version 4 (released in 2014) also introduced our line ratio method (Del Zanna et al. 2010) to adjust the relative degradation of many spectral lines. Differences between the various versions of the EVE MEGS calibration are significant (of the order of 50\%) at some wavelengths. However, the revised SEM irradiance values from Didkovsky \& Wieman (2014) remain in very good agreement with EVE MEGS and EVE ESP when the EVE v. 4 calibration is used, with some discrepancy during periods of increased activity, when the SEM 1 irradiances are about $10 \%$ higher. The generally good agreement with the EVE channels at a late stage in the SEM mission suggests that the SEM degradation is appropriately corrected.

For the actual comparison with NIS, we consider the Channels 1 and 3 SEM daily averaged count rates after the dark current counts had been subtracted. The observed count rates are compared with count rates that were modelled based on the spectra from NIS (discussed in Sect. 4) and the SEM effective areas (equal to the degradation-corrected SEM response function multiplied by the entrance aperture area). The effective areas used in the comparison include the additional off-band sensitivity determined from the 2007 SEM clone calibration and the most current degradation model derived using FISM reference spectra in Didkovsky \& Wieman (2014). Because the NIS comparison is based on the SEM observed count rates not the irradiance data product, it does not depend on the reference spectra adopted for SEM data processing beyond the modest influence that the reference spectrum has on the SEM degradation model as indicated above.

The NIS full-Sun scans were obtained in about $13 \mathrm{~h}$, so the resulting irradiances are not strictly simultaneous with the SEM measurements. However, we checked that daily SEM 1 variations for the selected dates are small, a few percent at most. The 46 NIS dates of the NIS observations were in fact selected during periods of relatively small solar variability.

We also looked at the SEM high time cadence (15 s) data for many of the days around the Solar Cycle 23 maximum and for days during the following minimum. The variations over the daily averages for Channels 1 and 3 are only a couple of percentage points or less and are not significantly higher for the solar maximum days than they are during solar minimum. Therefore, the effects of observed small solar flares for the dates considered are very small.

\section{Modelling the SEM 1 count rates (CDS+DEM)}

It turns out that a significant contribution to the SEM 1 count rates comes from coronal lines, most of which are not observed by NIS. We estimated them with differential emission measure (DEM) modelling. We used the Del Zanna (1999) method, where the DEM is a spline distribution, along with manual selection of the spline nodes.

The majority of the strongest spectral lines in the SEM band have contribution functions $G(T)$ that are largely independent of the density. Thus the precise value of the adopted value of density would not affect the results. A simple model with a constant density of $3 \times 10^{8} \mathrm{~cm}^{-3}$ was adopted for the inversion (DEM) and forward modelling.

It is well known that solar coronal abundances present variations and differ from the photospheric ones. The ratio of the abundances of the low $(\leq 10 \mathrm{eV})$ first ionization potential (FIP) elements vs. the high-FIP ones is generally thought to be higher in coronal plasma. However, we found that the CDS irradiance observations are consistent with the Asplund et al. (2009) photospheric abundances, which we adopt here. We note that the $\mathrm{Ne}$ abundance recommended by Asplund et al. (2009) is not obtained from photospheric measurements, but rather from coronal measurements.

The low-T part of the DEM is well constrained by NIS lines from high-FIP $\mathrm{O}$ and $\mathrm{Ne}$, while the high- $T$ part (above $0.8 \mathrm{MK}$ ) is constrained by lines from low-FIP elements, such as $\mathrm{Mg}, \mathrm{Fe}$, and $\mathrm{Si}$. One example that is typical of low solar activity is shown in Fig. 1 (top). The boxes are plotted at the $\operatorname{DEM}\left(T_{\text {eff }}\right) \times$ the ratio of the observed vs. predicted irradiance, where $T_{\text {eff }}$ is the effective temperature

$T_{\text {eff }}=\int G(T) \operatorname{DEM}(T) T \mathrm{~d} T / \int G(T) \operatorname{DEM}(T) \mathrm{d} T$,

i.e. an average temperature, weighted by the DEM distribution. Here, $T_{\text {eff }}$ is often quite different from $T_{\max }$, the temperature where the contribution function $G(T)$ of a line has a maximum. The DEM modelling reproduces the irradiances of the strongest lines to within 10-20\%.

During increased solar activity, active regions produce some additional plasma in the $1-3 \mathrm{MK}$ range, but most notably a secondary peak in the DEM around $3 \mathrm{MK}(\log T=6.5)$, as shown in Fig. 1 (bottom). The abundances of the low-FIP elements in 

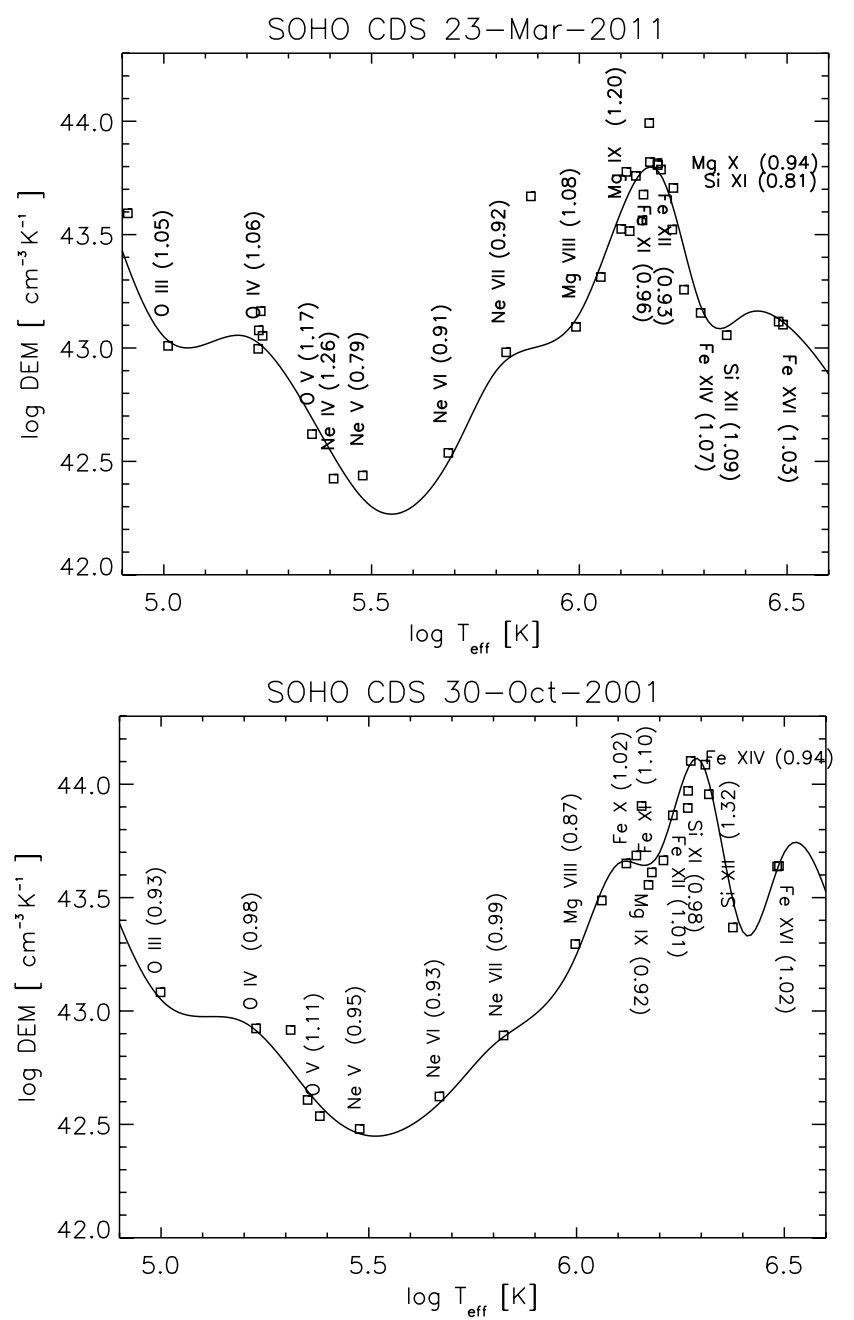

Fig. 1. Top: DEM distribution for 23 Mar. 2011, representative of a period of relatively low solar activity. Bottom: the DEM distribution for the 30 Oct. 2001, representative of a period of relatively high solar activity. The points are displayed at the effective temperature (see text). The labels indicate the main ion and the ratio of the predicted vs. observed irradiance.

the 3 MK active region plasma are approximately enhanced, compared to those of the low-FIP elements, by a factor of 3 as discussed in Del Zanna (2013), Del Zanna \& Mason (2014). DEM modelling of the EUV irradiances should therefore in principle be done by varying the abundances of the low-FIP elements with the temperature. However, in this paper we are not interested in the physical interpretation of the DEM but merely use the DEM to model the coronal spectrum for the SEM 1 band, so we have kept the abundances fixed to the photospheric values.

We then used the DEM distributions to calculate the predicted irradiances in all the lines contributing to the SEM 1 band. We replaced the He II $304 \AA$ and Si XI $303 \AA$ irradiances with those measured by NIS, and then folded the synthetic spectra with the SEM effective areas (which we recall include the current understanding of the SEM degradation). We obtained SEM 1 synthetic spectra, such as those shown in Fig. 2.

The synthetic spectra during low solar activity clearly indicate that the He II $304 \AA$ contributes a significant fraction of the total count rates, although the Fe XV $284.1 \AA$ line by itself provides a significant contribution, being close to the peak of the SEM 1 effective area. NIS observes strong lines from nearby ionization stages (Fe XIV and Fe XVI), as well as the
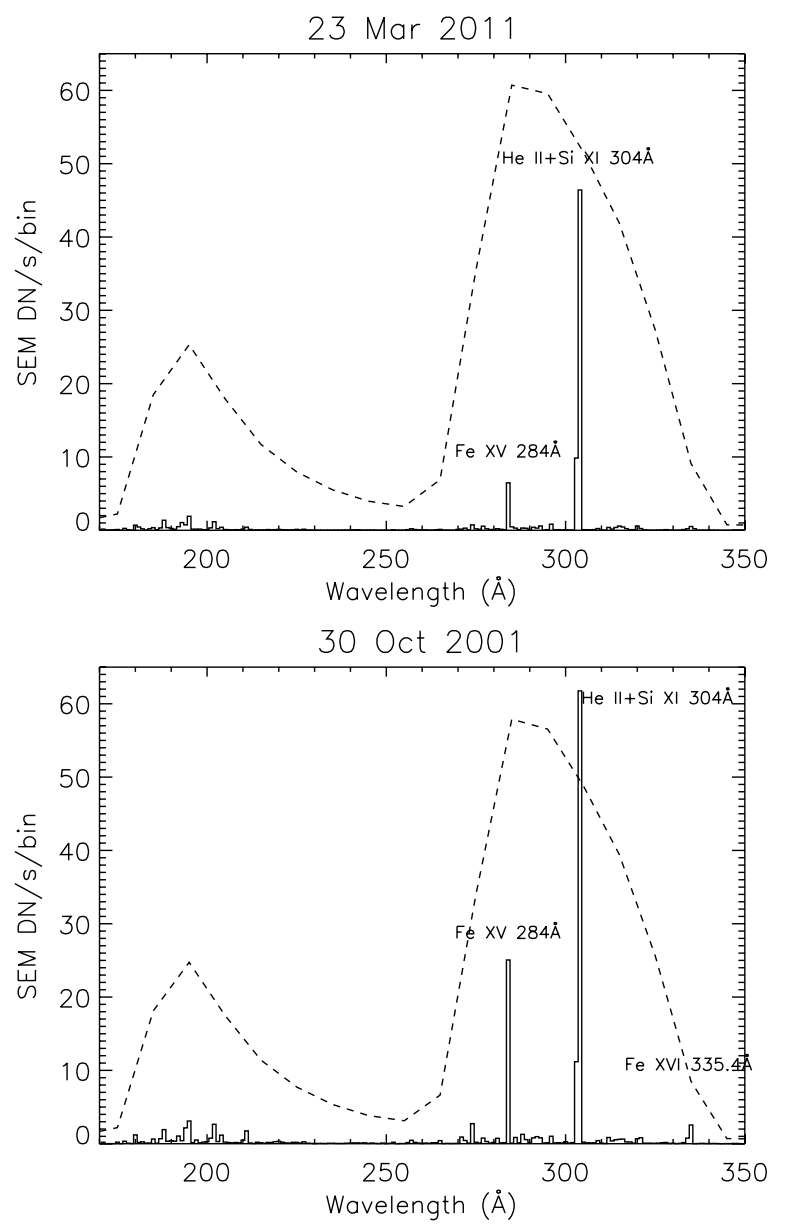

Fig. 2. SEM 1 synthetic spectra corresponding to the two selected dates, 23 Mar. 2011 and 30 Oct. 2001, representative of low and high solar activity, obtained from the CDS+DEM method. The dashed line indicates the shape of the SEM 1 effective area.

Si XII $520.6 \AA$ line, which is formed over a similar temperature to Fe XV. Therefore, the irradiance of the Fe XV $284.1 \AA$ line should have a small uncertainty (within 10\%). Indeed, we found excellent agreement between the predicted irradiance and the one measured on the same day (23 Mar. 2011) by the EVE calibration rocket, as Fig. 3 shows. There is also good agreement between the predicted irradiances of all the strongest coronal lines and those actually observed by EVE MEGS, confirming that the CHIANTI database is now relatively complete for these lines. Overall, the total count rates that were modelled based on the spectrum from the NIS observations ( $97 \mathrm{DN} / \mathrm{s}$ ) are in excellent agreement with those modelled based on the EVE spectrum (110 DN/s), as well as with the actual SEM 1 measured count rates $(92 \mathrm{DN} / \mathrm{s})$.

The synthetic spectra during high solar activity (Fig. 2, bottom) clearly show that the He II $304 \AA$ typically contributes about $40 \%$, while the Si XI $303 \AA$ and Fe XV 284.1 $\AA$ lines alone contribute about $20 \%$ during solar maximum. The remainder (about $40 \%$ ) is due to all the other lines, mostly coronal ones (i.e. formed around 1-3 MK).

To further assess the reliability of the CDS+DEM method during periods of increased activity, we searched the CDS database for all the USUN observations during the highest solar activity, when SDO/EVE was observing. We selected the 2012 April 19. Figure 4 shows a zoom-in of the SEM 1 spectra, as estimated from the CDS+DEM method and the 
G. Del Zanna et al.: The EUV spectrum of the Sun: SOHO, SEM, and CDS irradiances
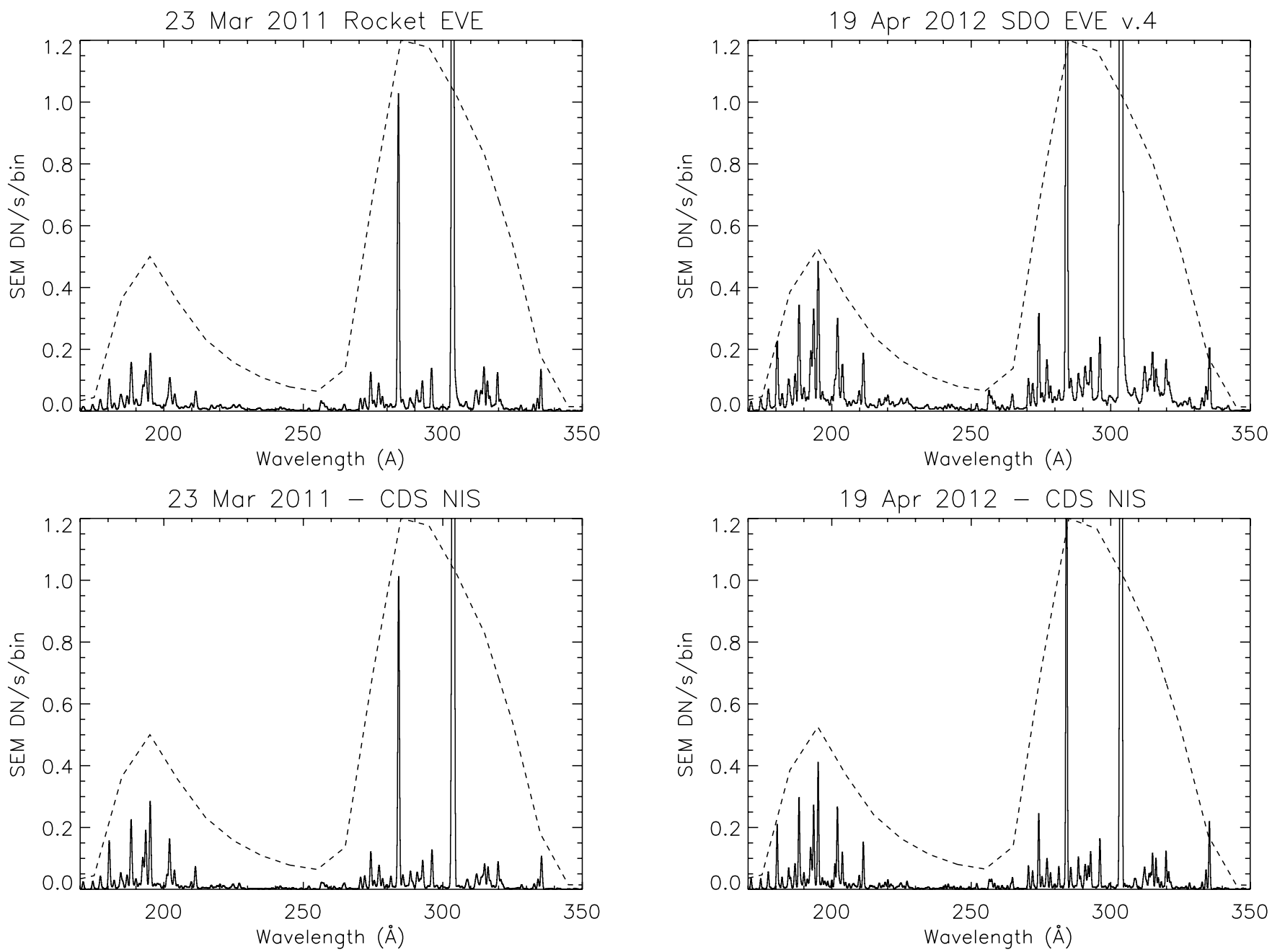

Fig. 3. SEM 1 synthetic spectra for 23 Mar. 2011, as obtained from the SDO EVE MEGS calibration rocket and from CDS/NIS. The dashed line indicates the shape of the SEM 1 effective area.

SDO/EVE v.4 observed spectra. Good overall agreement can be seen, although the intensities of some emission lines could be improved further. Differences in single lines in some sense average out, providing excellent agreement between the total count rates modelled based on the CDS+DEM method $(110 \mathrm{DN} / \mathrm{s})$ and those modeled based on the EVE MEGS v.4 data (107 DN/s).

We repeated the DEM analysis and forward modelling for all the NIS full-Sun observations. Interestingly, the DEM of the transition region (up to about $0.8 \mathrm{MK}$ ) does not change significantly over the solar cycle. This is ultimately because transition region lines vary little during the solar cycle, as shown for the first time in Del Zanna \& Andretta (2011) and explained by Andretta \& Del Zanna (2014).

Figure 5 shows the SOHO SEM 1 observed count rates, compared to those predicted by CDS. Very good agreement is found during solar minimum conditions, confirming the agreement in the calibration of the He II $304 \AA$ line. We expect agreement during later dates, given that our CDS irradiances agree with those from SDO EVE (as discussed in a forthcoming paper), and the SEM 1 observations also agree with SDO EVE MEGS (as we pointed out previously). On the other hand, the SEM count rates are consistently higher during the previous solar maximum between 1998 and 2005, by about 30\%. As an index of the solar activity, we also plot in Fig. 5 the F10.7 radio flux for the selected dates.

Fig. 4. SEM 1 synthetic spectra for 19 Apr. 2012, representative of moderate solar activity, as obtained from the SDO/EVE MEGS v.4 spectra (top) and the CDS/NIS (bottom). The dashed line indicates the shape of the SEM 1 effective area.

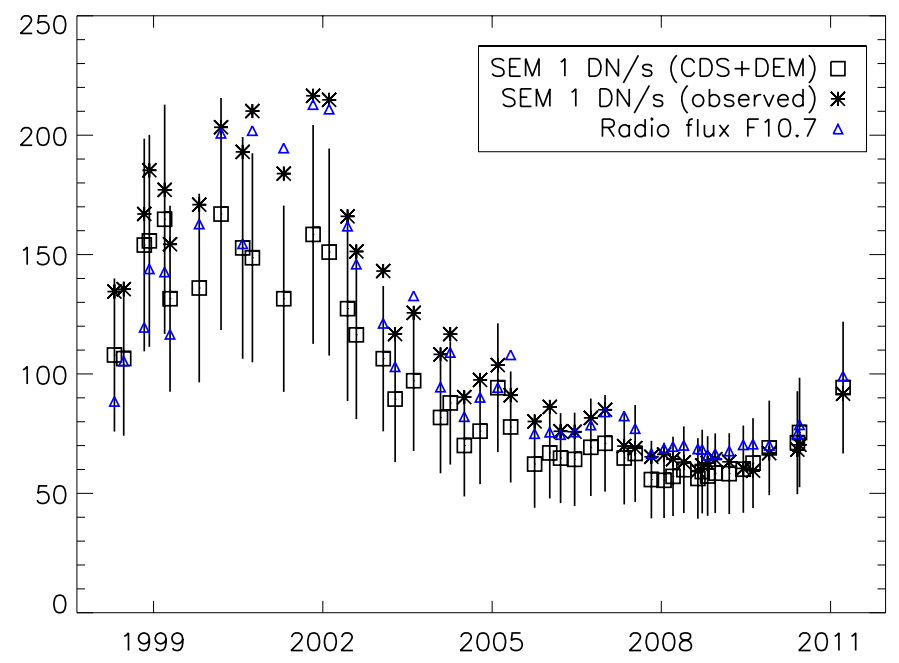

Fig. 5. SOHO SEM 1 observed count rates vs. those predicted using the CDS+DEM method. Estimated uncertainties on the predicted values are $30 \%$. The F10.7 radio flux for the corresponding dates is also shown.

Figure 6 shows the percentage contributions to the SOHO SEM 1 observed count rates for the selected dates. It confirms that the coronal line contribution to the SEM 1 band is 


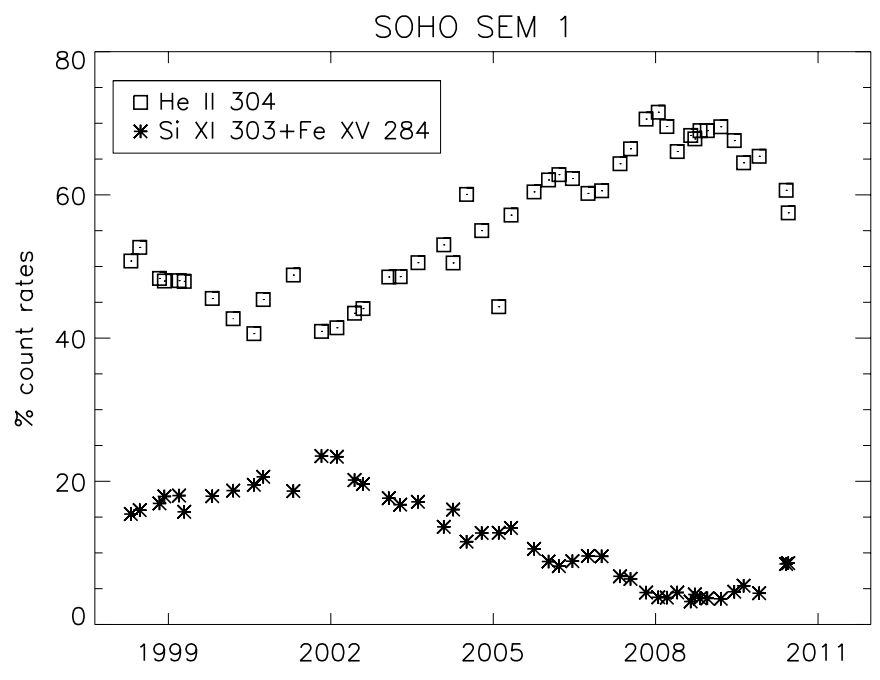

Fig. 6. Percentage contributions to the observed line count rates in the SOHO SEM 1, as estimated with the CDS+DEM method.

significant throughout the solar maximum. The Si XI $303 \AA$ and Fe XV 284.1 A lines alone contribute about $20 \%$.

The significant change in the EUV spectrum of the Sun from minimum to maximum conditions clearly affect the calibration of the SEM 1 channel, especially now that we are aware of the larger contribution from the coronal lines in the SEM 1 off-band. These coronal lines increase significantly during solar maximum, as described in detail in Del Zanna \& Andretta (2011).

FISM provides a set of historical time-varying reference spectra for the SEM calibration. However, FISM is a model and not an actual observation. FISM models the EUV irradiances with a semi-empirical approach, by using proxies to estimate the EUV variability in specified wavelength bins. The FISM model is based on the TIMED SEE EGS (Woods et al. 2005) measurements, which are not as accurate as those from SDO/EVE MEGS, for example. To check how accurate FISM reproduces observed irradiances and how significant differences are for the SEM calibration, we considered a few dates and made some comparisons between modelled and observed irradiances.

Two examples are shown in Fig. 7. The SOLERS22 is clearly discrepant, while the differences between FISM, CDS+DEM, and EVE MEGS, although significant in some wavelength ranges, overall produce small differences in the SEM 1 irradiance, when the spectra are used as references. The total SEM 1 photon flux $\left(10^{8}\right.$ phot $\left.\mathrm{cm}^{-2} \mathrm{~s}^{-1}\right)$ for 31 May 2010 is 87 using the CDS+DEM modelled irradiances, 90 using EVE MEGS, 93 using FISM, and 101 using SOLERS22. For 30 Oct. 2001, the total SEM 1 photon flux is 279 using CDS+DEM, 271 using FISM, and 292 using SOLERS22. The above SOLERS22-based SEM 1 flux values account for the aforementioned off-band responsivity discovered in the 2007 SEM clone calibrations and thus are not the same as SEM version 3.1 flux values, which use SOLERS22 but do not account for this additional responsivity.

\section{Conclusions}

Most historical measurements of the strongest EUV line, the He II $304 \AA$, have turned out to be incorrect by large factors. With a revised CDS calibration, excellent agreement with EUNIS and SDO EVE MEGS was found (Del Zanna \& Andretta 2011).
In this paper, we also showed excellent agreement, within a relative $10 \%$ or so, between the CDS and SOHO SEM 1 observations during solar minimum conditions. This is a remarkable achievement, considering that notoriously cross-instrument comparisons in the EUV have rarely shown relative agreement to within $30 \%$ (Pauluhn et al. 2002).

We cannot find any obvious reason why the SEM 1 observed count rates are consistently higher than the estimated ones during increased solar activity in 1998-2005 (the 10.7 radio flux for these dates ranged from 80 to 220, see Fig. 5). We see no reason why the CDS calibration of the He II $304 \AA$ would be at fault before 2005 , especially considering that the irradiance ratio of this line with the He I $584 \AA$ is constant in time (Del Zanna \& Andretta 2011).

About $50 \%$ to $60 \%$ of the count rates in the SEM 1 during increased solar activity come from coronal lines, so a plausible explanation is that their contribution to the SEM 1 is underestimated. This could in principle occur if the wavelengthdependent degradation of the SEM 1 during the first years of the mission was not as modelled. However, the SEM degradation was revised using the FISM modelled irradiances, which seem accurate enough for this purpose, as we have shown. If the SEM degradation does not correctly track the evolution of the sensitivity profile of the on-orbit instrument (i.e. the shift in relative sensitivity from longer to shorter wavelengths with time), we would expect that this would produce a long-term trend in the SEM calculated irradiances that would be evident in comparisons with solar indices and with our sounding rocket measurements. However, we do not see any obvious trend in such comparisons.

The contribution of the coronal lines could in principle by the DEM modelling, because the CHIANTI database is still not complete in the EUV or because of the various uncertainties related to the method, the varying iron abundance, etc. However, we see no obvious reasons why such uncertainties would provide a consistent shift. Furthermore, direct comparisons between modelled irradiances and those observed by SDO/EVE during increased activity (2012 April 19), when the radio F10.7 flux was 139 show good agreement (Fig. 4). We cannot rule out problems in our modelling for even more active conditions; however, for a significant fraction of the dates within 1998 and 2005, the 10.7 radio flux was below 139 , and the discrepancy is still there.

It is known that energetic particle events (such as those observed with the GOES proton monitor) increase the SEM count rates at about the same time (see, e.g. Didkovsky et al. 2007). However, except for isolated cases (e.g. 2001 October 30) the GOES proton fluxes are nominally at background levels around the dates we have considered here for the comparison. The SEM DN have therefore not been corrected for such contamination. Clearly, the background level of the energetic particles vary with the solar cycle. The fact that during increased solar activity the observed SEM 1 count rates are about $10 \%$ higher than those estimated from the SDO EVE MEGS suggests that part of the discrepancy between SEM and CDS might be ascribed to the effects of the energetic particles. To find a satisfying explanation for the discrepancy will require further investigation.

Acknowledgements. G.D.Z. acknowledges support from SOLID. SOLID (First European SOLar Irradiance Data Exploitation) is a collaborative SPACE Project under the Seventh Framework Programme (FP7/2007-2013) of the European Commission under Grant Agreement No. 313188. G.D.Z., S.R.W., and V.A. acknowledge support for participating in two workshops on the intercalibration of EUV instruments held at the Royal Observatory of Brussels, partially funded by 
G. Del Zanna et al.: The EUV spectrum of the Sun: SOHO, SEM, and CDS irradiances
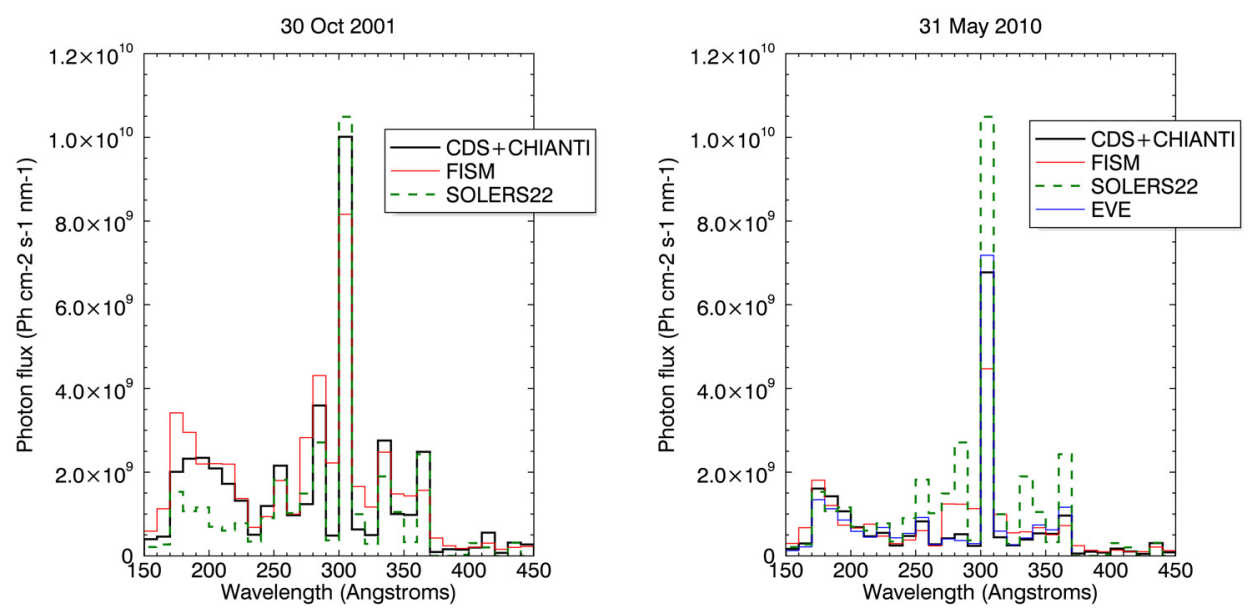

Fig. 7. Spectral irradiances (in photon flux units) within the SEM 1 bandpass for two dates (during maximum in 2001 and minimum activity in 2010) based on the SOHO CDS NIS + DEM approach are compared to those estimated by FISM, by SOLERS22, and for the 2010 case to those observed by SDO/EVE MEGS. When used as reference spectra for determining irradiance from the SEM 1 observed count rates, the CDS NIS + DEM, FISM, and SDO/EVE spectra yield similar results, whereas SOLERS22 produces SEM irradiances that are systematically higher.

STCE. V.A. acknowledges partial support by the Italian Space Agency (ASI), through ASI-INAF contract I/013/12/0. SOHO is a mission of international cooperation between ESA and NASA. CDS was built and operated by a consortium led by the Rutherford Appleton Laboratory (RAL), which includes UCL/Mullard Space Science Laboratory, NASA/ Goddard Space Flight Center, Max Planck Institute for Extraterrestrial Physics, Garching, and Oslo University.

\section{References}

Andretta, V., \& Del Zanna, G. 2014, A\&A, 563, A26

Asplund, M., Grevesse, N., Sauval, A. J., \& Scott, P. 2009, ARA\&A, 47, 481

Brekke, P., Thompson, W. T., Woods, T. N., \& Eparvier, F. G. 2000, ApJ, 536, 959

Chamberlin, P. C., Hock, R. A., Crotser, D. A., et al. 2007a, SPIE Conf. Ser., 6689

Chamberlin, P. C., Woods, T. N., \& Eparvier, F. G. 2007b, Space Weather, 5, 7005

Del Zanna, G. 1999, Ph.D. Thesis, Univ. of Central Lancashire, UK

Del Zanna, G. 2013, A\&A, 558, A73

Del Zanna, G., \& Andretta, V. 2011, A\&A, 528, A139

Del Zanna, G., \& Andretta, V. 2015, A\&A, submitted

Del Zanna, G., \& Mason, H. E. 2014, A\&A, 565, A14
Del Zanna, G., Bromage, B. J. I., Landi, E., \& Landini, M. 2001, A\&A, 379, 708 Del Zanna, G., Andretta, V., Chamberlin, P. C., Woods, T. N., \& Thompson, W. T. 2010, A\&A, 518, A49

Del Zanna, G., Dere, K. P., Young, P. R., Landi, E., \& Mason, H. E. 2015, A\&A, in press, DOI: 10.1051/0004-6361/201526827

Didkovsky, L., \& Wieman, S. 2014, J. Geophys. Res. (Space Phys.), 119, 4175

Didkovsky, L. V., Judge, D. L., Jones, A. R., Wieman, S., \& Tsurutani, B. T. 2007, Astron. Nachr., 328, 36

Didkovsky, L. V., Judge, D. L., Wieman, S. R., \& McMullin, D. 2010, in SOHO23: Understanding a Peculiar Solar Minimum, eds. S. R. Cranmer, J. T. Hoeksema, \& J. L. Kohl, ASP Conf. Ser., 428, 73

Harrison, R. A., Sawyer, E. C., Carter, M. K., et al. 1995, Sol. Phys., 162, 233

Hock, R. A., Chamberlin, P. C., Woods, T. N., et al. 2009, Sol. Phys., in review

Pauluhn, A., Huber, M. C. E., \& von Steiger, R. 2002, The Radiometric Calibration of SOHO ISSI Scientific Series, SR-002

Wang, T., Thomas, R. J., Brosius, J. W., et al. 2011, ApJS, 197, 32

Wieman, S. R., Didkovsky, L. V., \& Judge, D. L. 2014, Sol. Phys., 289, 2907

Woods, T., Ogawa, H., Tobiska, K., \& Farnik, F. 1998, in Solar Electromagnetic Radiation Study for Solar Cycle 22, eds. J. M. Pap, C. Frohlich, \& R. K. Ulrich (Dordrecht, Netherlands: Kluwer), 511

Woods, T. N., Eparvier, F. G., Bailey, S. M., et al. 2005, J. Geophys. Res. (Space Phys.), 110, 1312

Woods, T. N., Eparvier, F. G., Hock, R., et al. 2012, Sol. Phys., 275, 115 